\title{
Ueber den peripheren Theil der Urwirbel.
}

\author{
Von \\ Med. Dr. Felix Ehrlich \\ in Wien. \\ (Aus dem Institute für Embryologie des Prof. Schenk in Wien.)
}

Hierzu Taf. XV. Fig. 1-4.

Durch die neueren Forschungen bezüglich des Verhaltens der einzelnen anatomisch bedeutenden Bestandtheile im Embryo der Wirbelthiere wurde dargethan, dass die Urwirbel bald nach ihrem Auftreten eine Reihe von Veränderungen durchmachen, welche der Differenzirung der Gewebe im Embryo vorhergehen. Zu diesen Veränderungen ist zunächst die Angabe Remak's ${ }^{1}$ ) zu zählen, nach welcher ein Urwirbel in drei Abschnitte von aussen nach innen zerfällt.

Der äusserste dieser Abschnitte diene als Grundlage für die Muskeln (Muskelplatte), aus dem mittleren gehen die Elemente des Knochensystems hervor und der innerste Abschnitt liefere die Grundlage für die Ganglien und speciell für die Intervertebralganglien.

Durch eine Reihe von Jahren war diese Lehre allgemein angenommen worden und schliesst sich ihr auch Kölliker ${ }^{2}$ ) in seiner Entwicklungsgeschichte an.

1) Remak, Untersuchungen über die Entwickelung der Wirbelthiere. Berlin 1855.

2) Kölliker, Entwickelungsgeschichte des Menschen und der höheren Thiere. Leipzig 1861. 
Wesentlich verändert wurde diese Angabe von Schenk ${ }^{1}$ ). Nach dieser unterscheidet man zunächst an jedem Urwirbel einen centralen und peripheren Theil. Der erstere wird allmälig massenhafter, wobei die Formation der Urwirbelmasse um die einzelnen in dem Embryo angelegten Höhlen vorgeschoben wird. Dieser Theil der Urwirbel wird, wie derselbe Forscher angiebt, zum Aufbaue von Knochen, Knorpeln, Muskeln und der übrigen Gewebe, welche um die angelegten Höhlen zu finden sind, verwendet.

Der periphere Theil der Urwirbel präsentirt sich als aus länglichen Zellen bestehend, welche in radiärer Richtung zum Kerne der Urwirbel stehen. Von diesem Theile berichtet Schenk weniger ausführlich.

0 ellacher ${ }^{2}$ ), der sich in seinen trefflichen Arbeiten über die Entwickelung der Knochenfische bezüglich der Verwendung des Kernes der Urwirbel den Angaben Schenk's anschliesst, ist der Meinung, dass aus dem peripheren Theile der Urwirbel möglicher Weise Horngebilde hervorgehen; lässt aber diese Angabe als unentschieden dahingestellt.

Götte ${ }^{3}$ ) zeigte bei Bombinator igneus, dass der periphere Theil der Urwirbel zu subcutanem Gewebe transformirt wird und lässt das metamorphosirte Gewebe nach unten gegen die Bauchfläche des Embryo mit der Chorda dorsalis sich vereinigen, während nach oben der periphere Theil der Urwirbel mit den umgebenden Gebilden des Centralnervensystems sich verbindet.

Bezüglich der Veränderangen des centralen Theiles der Urwirbel für Bombinator igneus schliesst er sich den Angaben Sch enk's und Oellacher's an. Diese Angaben bildeten den Ausgangspunkt für die vorliegenden Untersuchungen, welche ich hier folgen lasse. Wenn man an den Embryonen von künstlich gezüchteten Forellen (salmo fario) vom 30. Tage angefangen aufwärts, Querschnitte in der Höhe des Mitteldarmes anfertigt, so sieht man anfangs, dass der centrale Theil der Urwirbelmasse (U; Fig. 1) um die einzelnen

1) Schenk, Beitrag zur Lehre von den Organanlagen im motorischen Keimblatte. Wiener Sitzungsberichte der k. Akad. 1868.

2) Oellacher, Beitrag zur Entwicklungsgeschichte der Knochenfische. Leipzig 1872. Zeitschrift für wissenschaftliche Zoologie Bd. XXII und XXIV.

3) Götte, Ueber die Entwickelung des Bombinator igneus. Archiv für mikroskopische Anatomie Bd. V. 
angelegten Höhlen, die anf dem Querschnitte zu beobachten sind, sich ausgebreitet hat. Man sieht an dem vorliegenden Schnitte dieselbe zunächst um das Centralnervensystem (C), um die Chorda dorsalis (Ch), ferner um die Ausführungsgänge der Urnieren (Un) und endlich um den Darmkanal (D) gelagert.

Der periphere Theil der Urwirbel $\left(\mathrm{U}_{2}\right)$ dagegen umgiebt beiderseits längs des Embryonalleibes den Kern der Urwirbel.

Am Rückentheile des Embryo sehen wir die radiär stehenden Elemente des peripheren Theiles zu beiden Seiten des Nervensystems umbiegen und in der Richtung nach der centralen Fläche des Embryo ziehen. Hier kann man die Grenze des peripheren Theiles nicht genau bestimmen, es scheint, als würden die Elemente in jenen des Kernes der Urwirbel sich allmälig verlieren.

An der ventralen Seite sieht man das absteigende Stück des peripheren Theiles der Urwirbel vor dem Ausführungsgange des W olff'schen Körpers vorübergehen und in der Richtung gegen die breite Chorda dorsalis (Ch) ziehen. Hier verliert sich derselbe in ähnlicher Weise, wie an der Seite des Nervensystems im Kerne der Urwirbel. Wir sehen somit, dass der periphere Theil der Urwirbel, welcher anfangs den ganzen Urwirbel umgiebt, denselben in diesem Stadium nur nach aussen zu beiden Seiten des Embryo gegen das Nervenhornblatt begränzt, während die Innenseite des Kernes der Urwirbel dem Centralnervensysteme (C), der Chorda (Ch), dem Urnierengange (Un), der Aorta und der intermediären Zellenmasse von 0 ellacher ${ }^{1}$ ) anliegt.

In den weiter vorgerückten Stadien beobachtet man, dass die einzelnen Elemente des peripheren Theiles der Urwirbel ihre Form geändert haben. Während man in jüngeren Stadien den Längsdurchmesser der Zelle radiär zur Chorda stehen sah, beobachtet man, wie in Fig. 2 zu sehen ist, dass der Längsdurchmesser der einzelnen Elemente, die den peripheren Theil bilden, parallel zur Oberfläche des Embryo liegt. Die Elemente besitzen einen ovalen Kern und Kernkörperchen.

Nach innen vom peripheren Theile sieht man rundliche hellere Gebilde ( $r$ ), welche bereits dem Kerne der Urwirbel angehören.

Gegen die dorsale Seite zu findet man den Gebilden der Ur-

1) $1 . \mathrm{c}$. 
wirbel anliegend, die Elemente des peripheren Theiles dichter an einander gelagert, so dass man auf dem Querschnitte an dieser Stelle noch eine Verdickung desselben zu Gesicht bekommt.

An dem lateralen Theile des Embryo beobachtet man Einsenkungen (E Fig. 2) des peripheren Theiles der Urwirbel, welche in den Kern der Urwirbel hinein sich erstrecken und an einigen Stellen sogar den sceletogenen Theil der Chorda erreichen. Fig. 2 stellt zwei derartige Einsenkungen dar, wovon die eine $\mathrm{E}_{1}$ die Chorda erreicht, während die zweite sich im Kerne der Urwirbel verliert. Diese Einsenkungen bilden, wie man sich aus der Reihenfolge von Querschnitten überzeugen kann, zusammenhängende membranöse Scheidewände, welche einzelne Zellenmassen der Urwirbel zwischen sich fassen. Aus eben diesen Scheidewänden zwischen den Gebilden des Urwirbelkernes, welche anfangs aus zelligen Elementen zusammengesetzt sind, werden später Faserzüge, zum Theile auch Züge, welche homogen sind, so dass man genöthigt ist, anzunehmen, es seien sowohl die faserigen als auch die homogenen Züge aus den Elementen des peripheren Theiles der Urwirbel hervorgegangen.

Ueberdies beobachtet man an Fig. 2, dass jene Gebilde, welche zwischen der Chorda und dem Nervensystem nach aussen liegen (m), bereits knorpelig umgebildet sind. An manchen Präparaten beobachtet man nicht selten, dass die besprochenen Septa bis an diese knorpelige Zellenmasse reichen, die man als Anlage des bleibenden Wirbelkörpers anzusehen hat.

Die einzelnen Septa, welche zwischen den bereits zu Muskeln metamorphosirten Elementen des Kernes der Urwirbel liegen, zeigen eine Reihe von Fortsätzen, welche zu einer Vervielfältigung der Septa führen. Die dadurch entstandenen kleinen Septa bestehen aus äusserst feinen homogenen Zügen, welche an den Stellen, wo sie von den Hauptzügen abgehen, etwas dickwandiger sind; weiter entfernt davon aber verdünnt sich allmälig die Wandung und die Septa werden immer kleiner, bis sie in ihrem Umfange dem Umfange eines Muskelbündels gleichkommen.

Fig. 3, welche einem Querschnitte durch den Embryonalleib eines 10 T'age nach dem Ausschlüpfen aus der Fischale alten Forellenembryo entspricht, zeigt die weiteren Veränderungen des peripheren Theiles der Urwirbel. Aus ihr entnehmen wir eine Bestätigung für das Gesagte bezüglich der grösseren und kleineren Septa in den Gebilden des Kernes der Urwirbel. Zugleich werden 
wir anch sehen, dass die grösseren Faserzüge als Fascien zwischen der bei den Fischen verhältnissmässig stark ausgebildeten Rückenmuskulatur liegen, während die feineren homogenen Septa, welche von diesen abstammen, als umhüllende Gewebszüge für die Muskelfaserbündel dienen. Wir sehen an Fig. 3 den Querschnitt des Centralnervensystems (C), an welchem bereits in diesem Stadium das Cylinderepithel, die Ganglien der grauen Substanz und die marklosen Fasern der weissen Substanz zu beobachten sind. Unter demselben liegen die geschrumpfte Chorda (Ch), überdies Gefässlumina und Durchschnitte des Wolff'schen Körpers. Unterhalb dieser Gebilde ist der Querschnitt des Darmkanals (D) zu sehen, welcher an einem kurzen Mesenterium hängt.

Die Urwirbelmasse (U), welche zu beiden Seiten des Nervensystems und der Chorda liegt, ist von mehreren Septa (S) durchbrochen. Die Gebilde, welche zwischen den Septa liegen, lassen es deutlich erkennen, dass sie Querschnitte von Muskelbündeln sind, deren Fibrillen sich zumeist so anordnen, dass dieselben theils rings herum um eine centrale Höhlung stehen, theils hufeisenförmig gekrümmt sind - Fig. 4. Um diese Züge von Muskelfaserbündeln (Mf) gehen die kleinen homogenen Septa, welche auf dem Querschnitte ein bienenwabenähnliches Gefüge geben, in welchem die Muskelfaserbündel liegen. Die Wandung der einzelnen Fächer ist homogen und trägt zuweilen einen oder mehrere Kerne. Es scheint dieses Gewebe jenen Zügen zu entsprechen, welche Lö w ${ }^{\mathfrak{l}}$ ) neuerer Zeit um die Fibrillencylinder am ausgebildeten Muskel und den Sehnen beschrieben hat.

Die grösseren Faserzüge verschmelzen nach innen mit den faserigen Zügen, welche das Centralnervensystem umgeben (n). An den Stellen, wo dieselben theils unter einander zwischen den Muskeln sich verbinden, oder wo sich dieselben mit den umhüllenden Faserzügen des Nervensystems vereinigen, sind sie dicker, und ihre Faserzüge stehen dichter aneinander. An dem Schnitte (Fig. 3) beobachtet man noch seitlich eine spitz hervorragende Masse der Urwirbel, welche vom äusseren Keimblatte überzogen ist. Sie stellt uns die Anlage einer Flosse vom Forellenembryo dar. Nachdem sich der Seitenplattentheil des Embryo von beiden Seiten an der Bauchfläche vereinigt hat, kommt die seitlich gelegene Flosse wahr-

1) Löwe, Centralblatt für medicinische Wissenschaften, Berlin 1874. 
scheinlich an die Bauchfläche des Embryo zu liegen, und bildet in dieser Lage eine Bauchflosse.

Aus den Schilderungen an den oben erwähnten Präparaten geht nun zur Genüge hervor, dass dem peripheren Theile der Urwirbel bezüglich seiner Verwendung zum Aufbaue der thierischen Gewebe eine andere Bestimmung zukomme, als den Gebilden im Kerne der Urwirbel. Die Elemente im peripheren Theile der Urwirbel werden zu Bindegewebszügen metamorphosirt, die nicht nur die subcutanen Gebilde liefern (Götte), sondern auch als grössere Septa die Muskelmasse am Rücken des Wirbelthieres durchziehen und hier die Fascien bilden. Die kleineren Septa, welche von jenen ausgehen, dienen als fächerige Gebilde um die einzelnen Muskelfaserbündel.

\section{Erklärung der Abbildungen anf Taf. XV.}

Fig. 1-4.

C Centralnervensystem.

Ch Chorda dorsalis (durch Schrumpfung in ihrer Form geändert).

I intermediäre Schicht.

$\mathrm{U}_{1}$ Kern der Urwirbel.

$\mathrm{U}_{2}$ peripherer Theil der Urwirbel.

Un Urnierengang.

D Darm.

W Wolff'scher Körper.

m Knorpelmasse der Wirbelkörper.

N Gewebe aus den Gebilden des mittleren Keimblattes, welche das Centralnervensystem umgeben.

$E$ und $E_{1}$ Septa aus dem peripheren Theile der Urwirbel.

S Vereinigungsstelle dieser Septa.

$\mathrm{E}_{11}$ Extremität (Flosse).

Mf Muskelfaserbündel auf dem Querschnitte. 

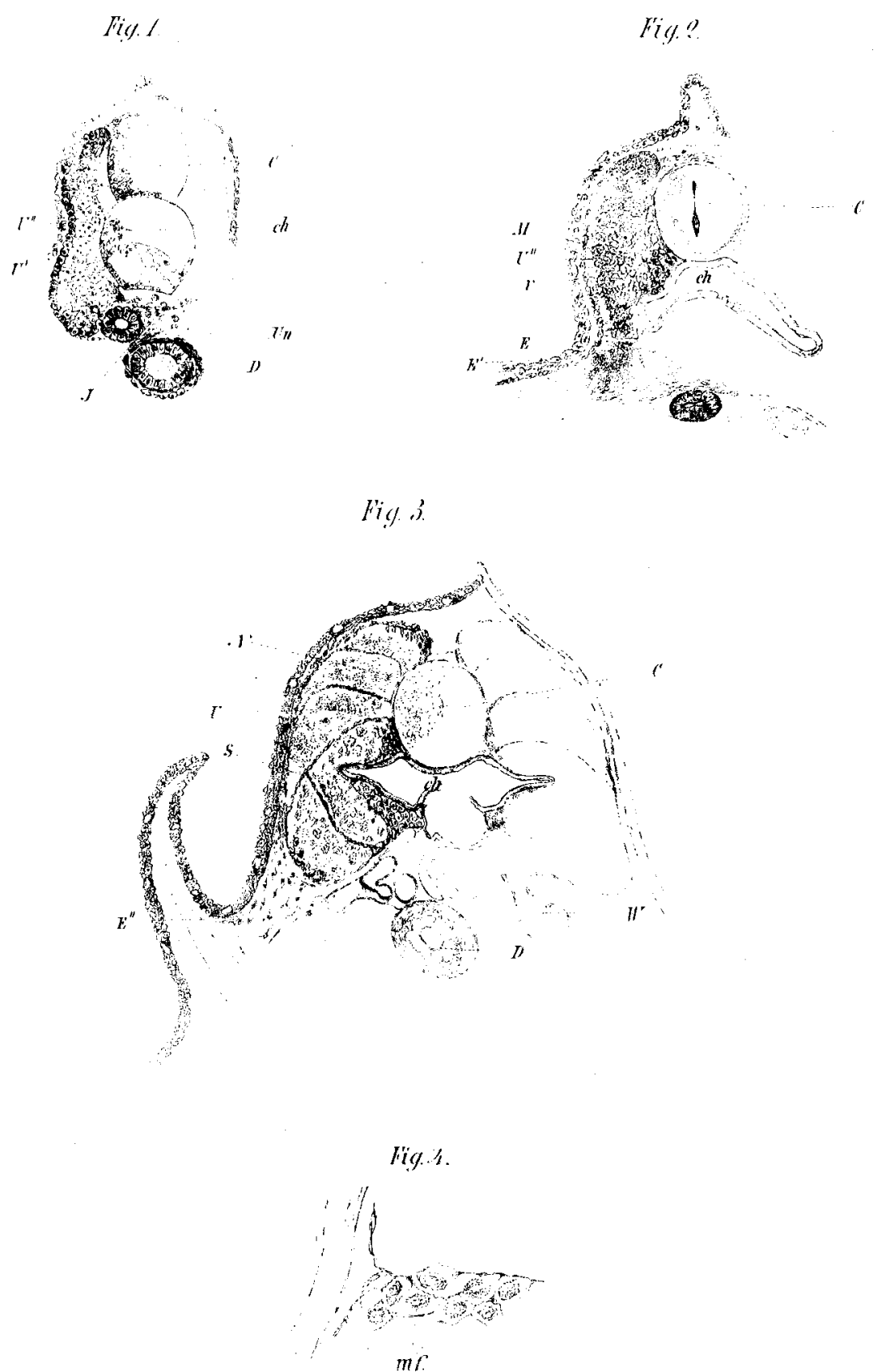